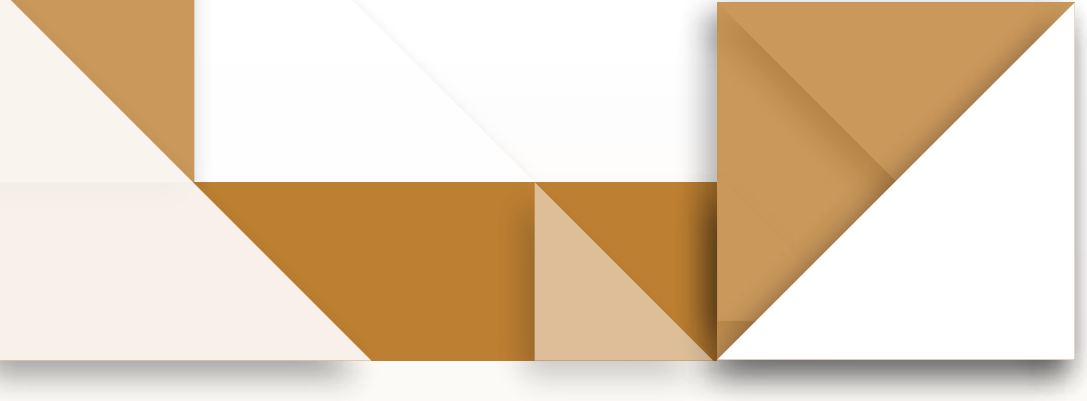

\title{
Fortalecimiento de la identidad profesional docente en el ámbito personal del profesor
}

\section{- Strengthening of Teachers Professional Identity in the Professor's Personal Field}

- Fortalecimento da identidade profissional
docente no domínio pessoal do professor

\section{Resumen}

En este artículo se presentan los resultados de una investigación con profesores en formación inicial para el fortalecimiento de la identidad profesional docente. Uno de los objetivos de la investigación estuvo relacionada con la exploración y desarrollo del ámbito personal del profesor para crear oportunidades de reestructuración de concepciones, actuaciones y actitudes acerca del sentido de ser profesor y de la importancia de su labor social y académica en la construcción de la ciudadanía de las actuales y futuras generaciones. Se analiza la incidencia de los cuatro ámbitos del conocimiento profesional del profesor (ACPP) (personal, conocimientos, práctico y contexto) en la IPD, haciendo relevancia en el ámbito personal. Mediante autobiografías, entrevistas y diarios personales; los nóveles, profesores expresan sentimientos y emociones sobre su pasado para intentar identificar el sentido que desde ese pasado dan al "yo" profesor. Se concluye que el proceso de reconfiguración de la Identidad Profesional Docente se dio al posibilitar experiencias para recrear momentos de su vida pasada y presente en los noveles profesores, y la forma cómo se proyectan hacia el futuro. En esta identidad se vinculan aspectos personales, motivaciones familiares, su sensibilidad con lo social y las relaciones que se pueden establecer con el momento socio-cultural vigente, creando un nuevo sentido de la profesión porque se conoce a sí mismo y sus alcances, convirtiéndose en un examinador crítico de la sociedad y del sistema educativo en particular, con miras a transformarlo desde lo que está a su alcance, que contribuye a analizar desde perspectivas distintas la profesión y en consecuencia sobre la identidad profesional docente.

Palabras clave

Identidad profesional; ámbito personal del profesor; formación inicial del profesorado

\section{Andrea Aristizábal Fúquene*}

Carmen Andrea Aristizábal Fúquene. Candidata a Doctora en Educación de la Universidad Distrital Francisco José de Caldas (die). Docente Adjunto de la Fundación Universidad Autónoma de Colombia. Bogotá-Colombia. Correo electrónico: andrea aristizabal@hotmail.com Orcid.org/0000-0003-2043-8686 


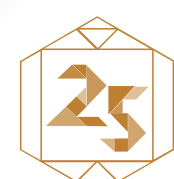

Años

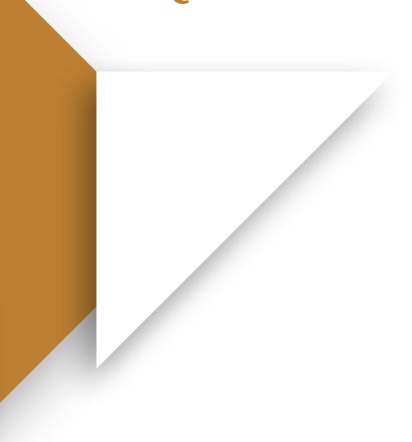

\section{Abstract}

This article presents the results of an investigation with professors in initial training to strengthen the teaching professional identity. One of the objectives of the research was related to the exploration and development of the personal field of the teacher to create opportunities for restructuring conceptions, actions and attitudes about the sense of being a teacher and the importance of his social and academic work in the construction of the citizenship of current and future generations. The incidence of the four fields of professional knowledge of the teacher (PKPC) (personal, knowledge, practice and context) in the IPD is analyzed, making relevance in the personal field. Through autobiographies, interviews and personal diaries, novice teachers express feelings and emotions about their past to try to identify the meaning that from that past they give to the "I" teacher. It is concluded that the process of reconfiguration of the Teachers Professional Identity was given by enabling experiences to recreate moments of his past and present life in the novice teachers, and the way they are projected into the future. This identity links personal aspects, family motivations, their sensitivity to the social and the relationships that can be established with the current socio-cultural moment, creating a new sense of the profession because they knows themselves and their scope, becoming a critical examiner of society and the educational system in particular, with a view to transforming it from what is within its reach, which contributes to analyze the profession from different perspectives and consequently on the teaching professional identity.

Keyword

Professional identity; personal field of the teacher; initial teacher training

\section{Resumo}

Este artigo apresenta os resultados de uma investigação com professores em treinamento inicial para fortalecer a identidade profissional do ensino. Um dos objetivos da pesquisa estava relacionado à exploração e desenvolvimento do campo pessoal do professor para criar oportunidades de reestruturação de concepções, ações e atitudes sobre o sentido de ser professor e a importância de seu trabalho social e acadêmico na construção da cidadania das gerações atuais e futuras. Analisa-se a incidência dos quatro campos do conhecimento profissional do professor (PCPC) (pessoal, conhecimento, prática e contexto) na DPI, tornando-se relevante no campo pessoal. Através de autobiografias, entrevistas e diários pessoais; os professores iniciantes expressam sentimentos e emoções sobre o passado para tentar identificar o significado que desse passado dão ao "eu" professor. Conclui-se que o processo de reconfiguração da Identidade Profissional Docente foi realizado ao possibilitar experiências para recriar momentos de sua vida passada e presente nos professores iniciantes e a maneira como eles são projetados no futuro. Essa identidade vincula aspectos pessoais, motivações familiares, sua sensibilidade ao social e às relações que podem ser estabelecidas com o atual momento sociocultural, criando um novo sentido da profissão, porque conhece a si mesma e seu escopo, tornando-se um examinador crítico da sociedade e do sistema educacional em particular, com o objetivo de transformá-lo do que está ao seu alcance, o que contribui para analisar a profissão sob diferentes perspectivas e, consequentemente, na identidade profissional docente.

Palavras-chave

Identidade profissional; área pessoal do professor; formação inicial de professores 


\section{Introducción}

La identidad profesional docente no ha sido una característica relevante o que genere mayor interés en los procesos de investigación en la formación de profesores, puesto que se asume como algo que va implícito en esa formación. De acuerdo con el rastreo realizado, en las últimas dos décadas el concepto de identidad docente se ha convertido en un área importante de investigación y en evolución (Beijaard, Meijer y Verloop, 2004), así como en un área de investigación independiente (Bullough, 1997; Connelly y Clandinin, 1996; Knowles, 1992; Kompf, Bond, Dworet, y Boak, 1996). El concepto se refiere a la relación entre la vida y experiencias personales de los docentes y su vida profesional. Esta empieza a cobrar importancia dado que se cree que la Identidad Profesional Docente determina cómo enseñan los profesores, cómo se desarrollan profesionalmente y cómo se acercan a los cambios educativos (Schepens, Aelterman y Vlerickb, 2009).

\section{Referentes conceptuales de partida}

Algunos factores que han ido consolidando el estudio de la identidad profesional docente en la formación inicial de profesores denotan una mirada externa de lo que ha de entenderse como identidad profesional y de las condiciones que la regulan. No se identifican componentes personales a los que los docentes acuden para configurarla, ni se presentan sus características. La IPD se ha asumido como una condición implícita, inherente y natural de la formación y no como un proceso consciente y estructurado en los programas de licenciatura.

Así, Schepens, Aelterman y Vlerick (2009) sostienen que en la formación de profesores es crucial la identidad profesional y que en los planes de estudio de los programas de forma- ción docente se formulan unas competencias profesionales insuficientes para explicar la estructuración de esa identidad. Resaltan que las instituciones que forman profesores proporcionan conocimientos que a menudo son fragmentados y cuando se llega a la práctica, los docentes deben integrarlos por sí mismos, y en múltiples casos estos se muestran ineficaces para relacionar la teoría con la práctica. Para reducir estas deficiencias, plantean que la formación no solo debe centrarse en el cambio de comportamiento, las competencias - las creencias, sino también considerarse la identidad de los futuros docentes y su misión.

Con lo anterior, se refieren a interrogantes como: ¿̇ónde estoy? (entorno), ¿̇qué estoy haciendo? (comportamiento), ¿̇qué puedo hacer (capacidades y competencias), ¿̇qué creo? (creencias), ¿̇quién soy yo? (identidad) y żqué quiero? (misión).

Estos autores proponen un modelo de cebolla, como proceso para estructurar la identidad profesional docente, en el que los niveles internos y externos se influyen mutuamente. La identidad y la misión del profesor se ponen de relieve dado que constituyen el núcleo de la cebolla.

Por otra parte, se analiza cómo la política educativa influye en la identidad profesional docente. Al respecto, Gilmore, Hurst y Maher (2009) afirman que las reformas educativas en los países inciden de modo significativo en la identidad de los profesores quienes, para dar una respuesta exitosa a las reformas establecidas, requieren adecuar esa identidad a ellas. Es aquí donde la identidad puede ser construida por factores externos como lo es la política educativa, pero se desconocen los factores personales e internos que se relacionan con los externos.

Se rescata de su aporte lo que retoman de Luehmann (2007) quien describe cuatro 
procesos necesarios para el desarrollo de la identidad profesional docente. El primero implica la reflexión permanente sobre la experiencia con cuestiones de fondo de quién soy yo y quién quiero ser; el segundo, es el desarrollo que se da a través de las interacciones entre el individuo y el contexto de la experiencia en una comunidad de práctica; el tercero, es un proceso multifacético compuesto de sub-identidades que pueden responder a múltiples contextos; y, el cuarto, es el construido por la interacción con los alumnos a través del desarrollo de la confianza en sí mismos.

De acuerdo con lo anterior, se asume que la Identidad Profesional Docente es un proceso que conlleva diferentes transformaciones y estas no se dan de forma aislada. Por el contrario, el contexto influye de manera importante en su configuración. Esto denota su importancia para incluirla en el proceso de formación docente, puesto que las pasiones, compromisos, formas de actuar e interactuar, los valores y la moral influyen en su configuración (Luehmann, 2007) y, en efecto, en el éxito del ejercicio de la profesión.

Por su parte, Hong (2010) categoriza la IPD como un factor importante en la vida profesional de un docente. Esta es influenciada por la motivación, la eficacia, el valor de la autorrealización, el compromiso, la autoimagen, la autoestima, la actitud en el espacio laboral y la percepción hacia sí mismos. Triantafyllaki (2010) plantea que la IPD se da porque existe un cuerpo de conocimientos especializados que representan las características de un campo profesional $y_{\text {, }}$ dado que los profesionales se enfrentan a situaciones complejas e impredecibles, requieren una organización para que puedan aplicar ese conocimiento, además argumenta sobre la necesidad de su autonomía para hacer sus propios juicios. Esta autonomía es un componente esencial que actúa como una forma de responsabilidad compartida, por lo que se hace necesario explicitar los vínculos entre el conocimiento y la IPD.

Chong, Ling y Chuangoh (2011) están de acuerdo en que la identidad de los profesores está en constante cambio, desde que se opta por los estudios profesorales, dado que las imágenes desarrolladas desde su experiencia personal y sus estudios determinan una actitud hacia la enseñanza, su comprensión, sus creencias y su práctica. Estas imágenes son las que inciden en su ejercicio profesional y en su IPD. Para ello proponen como estrategia la reflexión permanente, dado que les permite dirigir de modo consciente el desarrollo de SU IPD, su inspiración y el entusiasmo por la profesión.

Tales escenarios de reflexión son posibles a través de una comunidad de práctica establecida desde su formación inicial, puesto que les permiten discutir, construir y adoptar posiciones de su identidad. Estos autores establecen que esas reflexiones y discusiones propician desarrollo profesional, por cuanto convertirse en un profesional implica realizaciones externas y personales, las cuales conllevan una reconfiguración de la IPD. 
Los argumentos descritos con anterioridad fueron fundamentales en la investigación, dado que se asume que los profesores en formación han de reconocer las realidades y tensiones educativas del contexto colombiano, de tal manera que se rechace o acepte lo que hasta ahora se ha estructurado, lo que significa ser profesor en unos escenarios particulares. De tal manera que, a partir de las prácticas discursivas que se realizan con los profesores en formación inicial, hagan frente a esas realidades de la cultura escolar y conviertan sus primeros años del ejercicio en una experiencia positiva, en la cual lo vivido durante su programa de formación les permita crecer y desarrollarse de modo constante. En consecuencia, se espera que estas experiencias vividas en su proceso de formación se combinen con sus creencias personales y experiencias previas para formar su identidad, que a su vez influirá en su futuro para la toma de decisiones.

Así, la intención de la investigación fue identificar y caracterizar el proceso de configuración y reconfiguración de la identidad profesional de docentes que trabajan en una Comunidad de Desarrollo Profesional (CODEP), propuesta inicial de García-Martínez e lzquierdo (2014), desde la exploración y desarrollo de los ámbitos del conocimiento profesional del profesor (ACPP).

Esta CODEP se concibió como un escenario conformado por profesores en formación inicial en el cual se propiciaron escenarios de integración, articulación y movilización de saberes propios de la docencia. Allí, a través de la interacción, discusión, reflexión, transferencia, colaboración y transformación de conocimientos y experiencias se le otorga un nuevo valor y sentido a la profesión docente. En estos procesos están implícitos los pensamientos, emociones y acciones que han de redefinir de modo continuo el ejercicio de la profesión, con miras a la cualificación perma- nente para mejorar la calidad de la educación en ciencias.

En cuanto al ámbito personal, se establece que está constituido por valores, creencias, actitudes e imágenes de sí mismo, la sociedad, la cultura y el tipo de ideologías que orientan el ejercicio profesional (Clarke y Hollingsworth, 2002). En este ámbito se considera la satisfacción de los profesores (Freeman, Ronald y Cimellaro, 2004), que puede estar determinada por el éxito en el rendimiento y desempeño de los estudiantes (Costa y Garmston, 1994; Glickman, 1990). Este ámbito se relaciona directamente con la identidad profesional por cuanto considera aspectos personales del profesor, de la imagen de sí mismo y su imagen en la sociedad.

\section{Metodología}

La investigación se centró en el paradigma cualitativo desde la perspectiva socio-crítica y naturalista-interpretativa; el grupo de trabajo se conformó con once profesores en formación, del programa de Licenciatura en química de la Universidad Distrital Francisco José de Caldas (Bogotá-Colombia), que iniciaban su práctica profesional docente. Se diseñó e implementó un modelo de intervención docente (Véase figura 1), con la aplicación de una serie de instrumentos en diversos momentos, para recopilar información de las experiencias vividas en diferentes escenarios y categorizarla según los ámbitos.

De igual manera, para las interpretaciones e inferencias sobre la configuración de la identidad profesional docente del grupo de profesores, se crearon dos matrices: la número $1(\mathrm{M} 1)$, corresponde a la síntesis de los fundamentos teóricos sobre los Ámbitos del Conocimiento Profesional del Profesor (ACPP) y su relación con la Identidad Profesional Docente (IPD) (Véase tabla 1), y la número 2 (M2), - 
que emerge de la número 1, en la que se precisan las categorías, subcategorías y atributos deseables de la IPD (Véase tabla 2). La estructura de la intervención realizada se resume en la figura 1 .

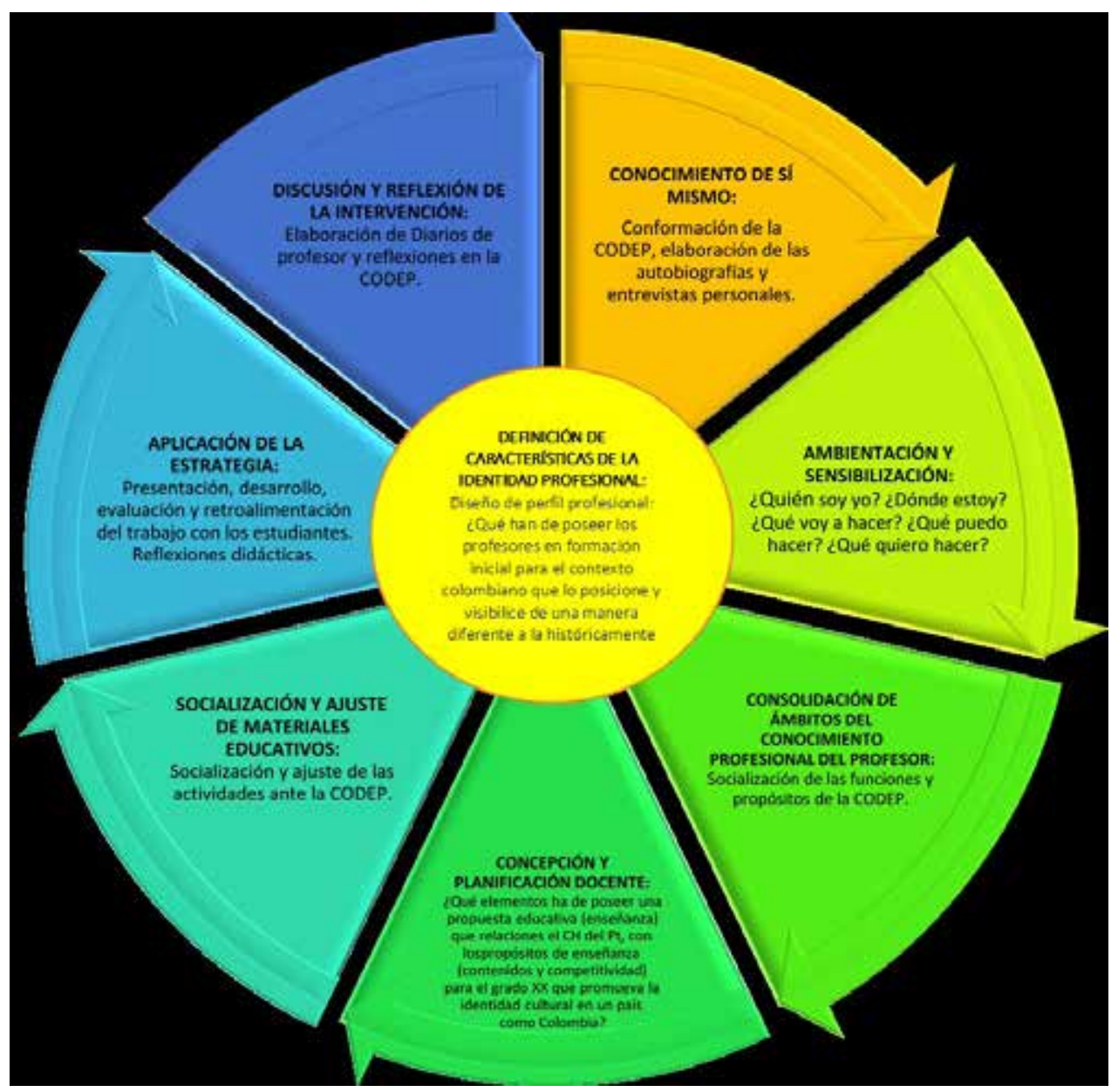

Figura 1. Modelo de intervención docente.

Fuente: elaboración propia 
Descripción de cada uno de los momentos de la intervención docente:

Tabla 1. Momentos de la intervención docente.

\begin{tabular}{|c|c|}
\hline Momentos & Acciones/Actividades \\
\hline $\begin{array}{l}\text { MOMENTO } 1 \\
\text { CONOCIMIENTO DE SÍ MISMO }\end{array}$ & $\begin{array}{l}\text { Conformación de la Comunidad de Desarrollo. } \\
\text { Elaboración de autobiografías (narrativa de expectativas personales de la profesión } \\
\text { previas, durante y futuras con el compromiso social de la profesión). } \\
\text { Entrevistas personales sobre las expectativas anteriormente mencionadas. }\end{array}$ \\
\hline $\begin{array}{l}\text { MOMENTO } 2 \\
\text { AMBIENTACIÓN } \\
\text { Y SENSIBILIZACIÓN }\end{array}$ & $\begin{array}{l}\text { Acercamiento al contexto socioeducativo (colegio) de los miembros de la CODEP. } \\
\text { Filmaciones y transcripciones de las discusiones y reflexiones en la CODEP en torno a: } \\
\text { ¿Quién soy yo? ¿Dónde estoy? ¿Qué voy a hacer? ¿Qué puedo hacer? ¿Qué quiero } \\
\text { hacer? }\end{array}$ \\
\hline
\end{tabular}

MOMENTO 3

CONSOLIDACIÓN DE LOS

ÁMBITOS DEL CONOCIMIENTO

Presentación de la propuesta investigativa en función de los ámbitos del conocimiento profesional del profesor.

PROFESIONAL DEL PROFESOR

Socialización de la misión, las funciones y propósitos de la CODEP.

\begin{tabular}{ll}
\hline & Socialización y discusión de los planes de estudios para los diferentes niveles de \\
& acuerdo con la normatividad (Estándares curriculares, Decreto 1290, PEI de la IED, Plan \\
& decenal $2006-2016$ y Cumbres Mundiales). \\
& Socialización y discusión del caso histórico del platino (Lectura general del caso. iQue \\
MOMENTO 4 & no te cuenten la Historia, reconstrúyela!). \\
DTAPA DE CONCEPCIÓN Y & Diseño de los materiales educativos bajo el siguiente núcleo problemático: ¿̇Qué \\
PLANIFICACIÓN DOCENTE & elementos ha de poseer una propuesta educativa (enseñanza) que relacione el caso \\
& histórico del platino con los propósitos de enseñanza (contenidos y competencias) \\
para el grado correspondiente, que promueva identidad cultural en un país como \\
Colombia?
\end{tabular}

Socialización, discusión y ajuste de la propuesta de enseñanza para los diferentes cursos.

MOMENTO 5

Preparación de las actividades por desarrollar con los estudiantes.

SOCIALIZACIÓN Y AJUSTE DE

Definición de las formas de evaluación y criterios de evaluación de las actividades MATERIALES EDUCATIVOS propuestas.

Socialización de las actividades ante la codep.

Ajuste de las actividades

\section{MOMENTO 6 \\ APLICACIÓN DE LA ESTRATEGIA}

APLICACIONN DE LAESTRATEGIA

Presentación de la propuesta de enseñanza a los estudiantes

Desarrollo de las actividades con los estudiantes

Evaluación de las actividades con los estudiantes

Retroalimentación del trabajo con los estudiantes

Autoevaluación de la estrategia a la luz de los ámbitos y de otros emergentes

\begin{tabular}{|c|c|}
\hline $\begin{array}{l}\text { MOMENTO } 7 \\
\text { SOCIALIZACIÓN, EVALUACIÓN Y } \\
\text { DISCUSIÓN DE LA APLICACIÓN DE } \\
\text { LA ESTRATEGIA }\end{array}$ & $\begin{array}{l}\text { Elaboración de los diarios de profesor en donde narre la experiencia vivida, descrita } \\
\text { desde cada uno de los ámbitos del conocimiento profesional. } \\
\text { Compartir en la CODEP la experiencia vivida. } \\
\text { Definición de aciertos, desaciertos y ajustes a las propuestas. }\end{array}$ \\
\hline $\begin{array}{l}\text { MOMENTO } 8 \\
\text { EVALUACIÓN DEL PROCESO DE } \\
\text { INTERVENCIÓN DOCENTE DESDE } \\
\text { LOS ÁMBITOS }\end{array}$ & $\begin{array}{l}\text { Una vez realizado este proceso, durante algunas semanas se identifica tanto el tipo } \\
\text { de interacciones como el de contribuciones que se suscitaron en la CODEP desde } \\
\text { cada uno de los ámbitos, que permitan definir algunas características de la identidad } \\
\text { profesional docente. } \\
\text { Se elaboran las reflexiones docentes producto de la experiencia vivida. }\end{array}$ \\
\hline $\begin{array}{l}\text { MOMENTO } 9 \\
\text { DEFINICIÓN DE LAS } \\
\text { CARACTERISTICAS DE LA } \\
\text { IDENTIDAD PROFESIONAL } \\
\text { DOCENTE EN PROFESORES }\end{array}$ & $\begin{array}{l}\text { Una vez socializada la experiencia vivida expresada de forma escrita (diario de } \\
\text { profesor) y de forma oral en la CODEP, cada uno de los profesores participantes } \\
\text { elabora un perfil de identidad profesional para profesores de química en formación } \\
\text { inicial. } \\
\text { Se socializa este perfil ante la CODEP. }\end{array}$ \\
\hline
\end{tabular}

Fuente: elaboración propia

Elaboración de los diarios de profesor en donde narre la experiencia vivida, descrita desde cada uno de los ámbitos del conocimiento profesional.

Compartir en la CODEP la experiencia vivida.

Definición de aciertos, desaciertos y ajustes a las propuestas.

Una vez realizado este proceso, durante algunas semanas se identifica tanto el tipo cada uno de los ámbitos, que permitan definir algunas características de la identidad profesional docente.

elaboran las reflexior

$$
\begin{aligned}
& \text { profesor) y de forma oral en la CODEP, cada uno de los profesores participantes } \\
& \text { elabora un perfil de identidad profesional para profesores de química en formación } \\
& \text { inicial. } \\
& \text { Se socializa este perfil ante la CODEP. }
\end{aligned}
$$


Por otra parte, para fines de interpretación de los resultados, en la tabla 2 se presentan los atributos deseables de la identidad profesional que se establecieron para el Ámbito personal.

Tabla 2. Atributos deseables de la identidad profesional en el Ámbito personal del Profesor.

\begin{tabular}{|c|c|c|c|c|}
\hline Ámbito & Categoría & Subcategoría & Cód. & Atributo \\
\hline \multirow{6}{*}{$\begin{array}{l}\text { Ámbito } \\
\text { personal }\end{array}$} & \multirow{6}{*}{$\begin{array}{l}\text { Conocimiento } \\
\text { de sí mismo }\end{array}$} & $\begin{array}{l}\text { Definición de sí } \\
\text { mismo }\end{array}$ & 1_Des & $\begin{array}{l}\text { Describe las características y cualidades } \\
\text { que tiene de sí mismo para ser profesor } \\
\text { de química. }\end{array}$ \\
\hline & & $\begin{array}{l}\text { Conocimiento } \\
\text { Unificado }\end{array}$ & 2_Narr & $\begin{array}{l}\text { Narra de manera oral y escrita hechos } \\
\text { y eventos de su vida pasada que } \\
\text { influenciaron e influencian su decisión } \\
\text { profesional como docente de química. }\end{array}$ \\
\hline & & \multirow{3}{*}{ Autodescubrimiento } & 3_Mani & $\begin{array}{l}\text { Manifiesta emociones cuando habla de la } \\
\text { profesión docente. }\end{array}$ \\
\hline & & & 4_Narra & $\begin{array}{l}\text { Narra cómo se imagina en su futuro } \\
\text { cercano y lejano en relación con sus } \\
\text { expectativas personales y el compromiso } \\
\text { social de la profesión docente. }\end{array}$ \\
\hline & & & 5_Nomb & $\begin{array}{l}\text { Nombra o enuncia características que } \\
\text { surgen, que reconoce o que se desarrollan } \\
\text { al transcurrir la Práctica Profesional. }\end{array}$ \\
\hline & & Influencia docente & 6_Mani & $\begin{array}{l}\text { Manifiesta cómo su profesor o } \\
\text { profesora de colegio les aportó o fue } \\
\text { gran influencia en la decisión de ser } \\
\text { licenciado en química. }\end{array}$ \\
\hline
\end{tabular}

Fuente: elaboración propia.

Para el proceso de análisis de la información, se empleó el software NVivo versión 10.0, programa con un potencial de tratamiento y organización de cantidades de información que se puede importar y organizar, en conjunto con relaciones y cruces de la misma que se estimen necesarios. En la investigación se delimitaron procesos y, en consecuencia, hubo reducción de datos. Fue indispensable esta reducción por el número de instrumentos aplicados, así como el de participantes. En efecto, se recurrió a gráficas de barras para el análisis e interpretación de esta información, así como para las inferencias de orden teórico relacionadas con este ámbito.

De acuerdo con lo anterior, la etapa de explotación de la información correspondió a los procedimientos manuales y los efectuados con el software NVivo 10.0 sobre las fuentes de información empleadas como las autobiografías, entrevistas y diarios de profesor. Se hizo de manera manual el análisis de contenido de las fuentes y se seleccionaron las Unidades de Registro (UR) que 
dieran cuenta de los atributos deseables establecidos en la matriz 2 y luego se realizaron operaciones de codificación en función de consignas llevadas a cabo previamente (Véase matriz 2: categorías, subcategorías y atributos deseables) importadas al software.

\section{Resultados}

A continuación, se presentan los resultados de los atributos deseables que más se fortalecieron en las autobiografías, entrevistas personales y diarios de profesor.

La figura 2, generada por el software Nvivo 10.0, presenta los atributos fortalecidos (características de IPD) en la autobiografía, de acuerdo con el número de unidades de registro identificadas (fragmentos de los discursos de los profesores seleccionados que dan cuenta de las características de la IPD).

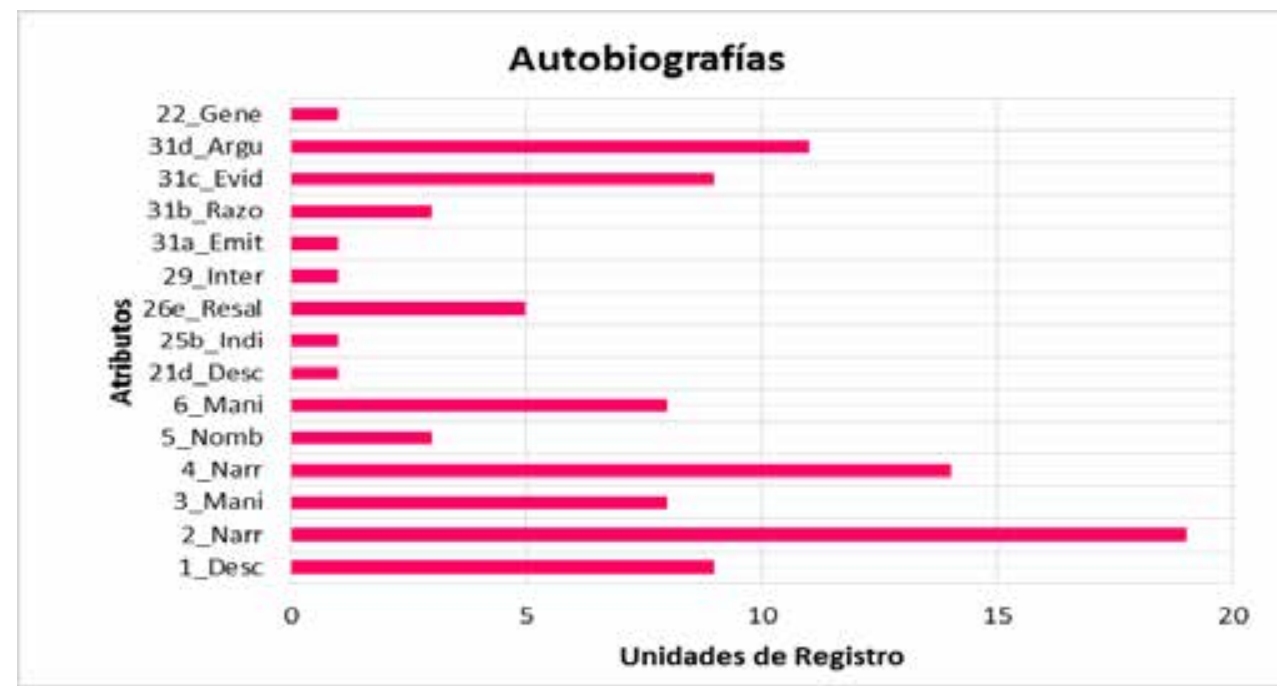

Figura 2. Atributos deseables de la Identidad Profesional Docente fortalecidos en la autobiografía.

Fuente: elaboración propia

En la gráfica, el eje Y corresponde a los códigos de los atributos deseables que más datos registraron en la autobiografía. El eje $X$ corresponde al número de unidades de registro (UR) los cuales son fragmentos, producto del análisis de contenido, que dan cuenta de los atributos de identidad profesional para el ámbito personal. Para este ámbito se establecieron seis atributos deseables, codificados de 1 a 6. Así mismo para las entrevistas (Véase figura 3) y los diarios de profesor (Véase figura 4). 


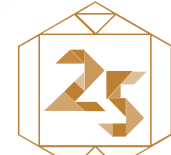

Años

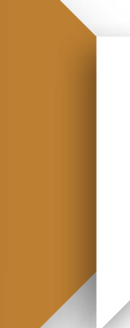

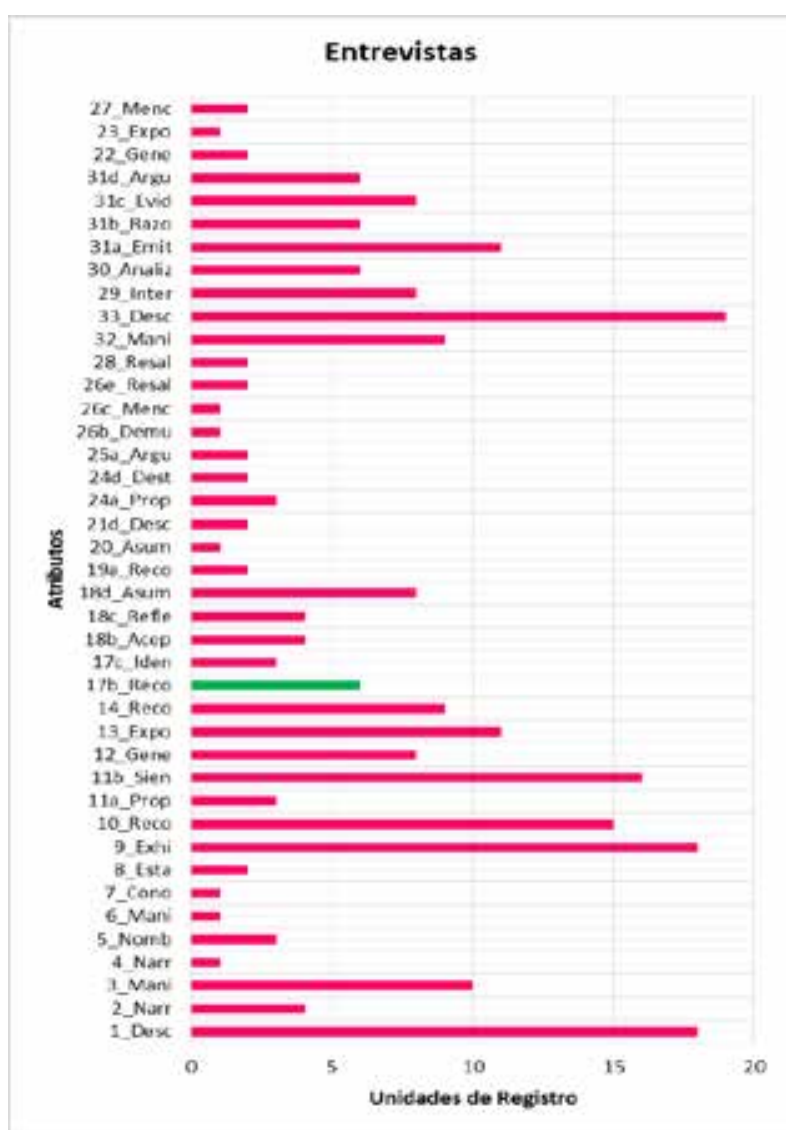

Figura 3. Atributos deseables de la Identidad Profesional Docente fortalecidos en la entrevista personal.

Fuente: elaboración propia

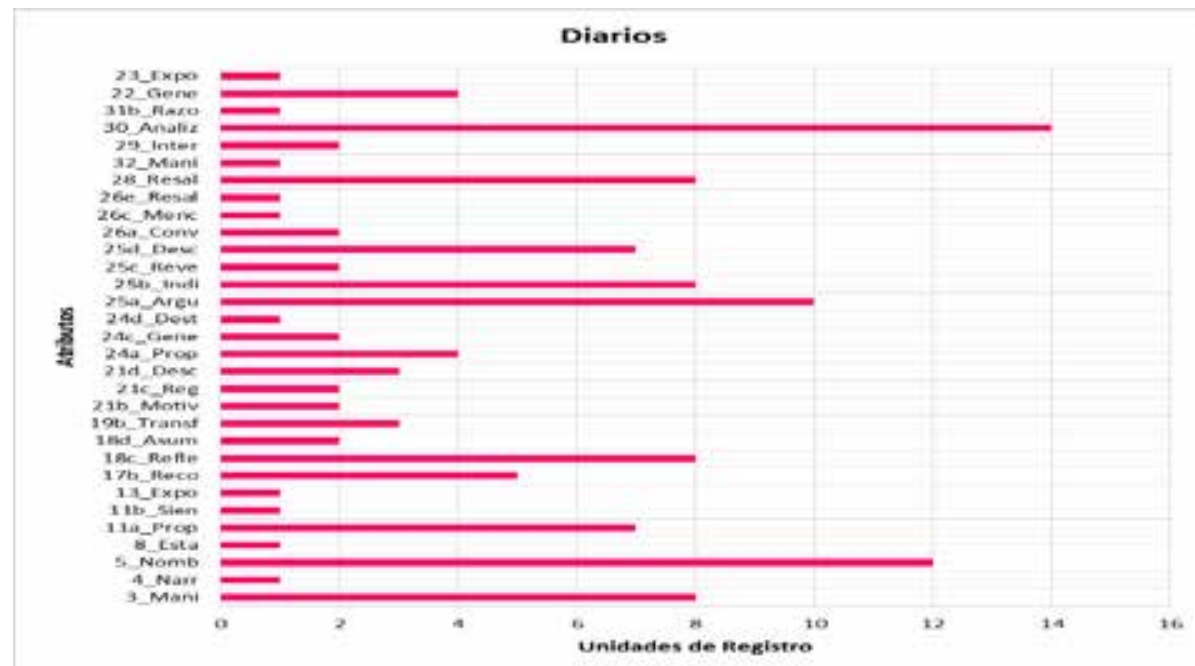

Figura 4. Atributos deseables de la Identidad Profesional Docente fortalecidos en el diario de profesor.

Fuente: elaboración propia 


\section{Discusión de resultados}

De acuerdo con los resultados obtenidos, se puede evidenciar la variedad y cantidad de atributos que se fortalecieron en los tres instrumentos. En la figura 2, correspondiente a las Autobiografías, los atributos que más se fortalecieron fueron el 2_narr y 4 _narra. En las unidades de registro que se seleccionaron, se evidenció que las personas construyen su identidad haciendo un relato, que no solo es un recorrido del pasado sino un modo de recrearlo, en un intento por descubrir un sentido e inventar el yo. Supone un ejercicio reflexivo de autodescubrimiento del significado que han tenido los acontecimientos y experiencias que han jalonado su vida.

Este instrumento fue fundamental puesto que los docentes en formación no habían tenido esta experiencia, no habían realizado reflexiones intencionadas sobre sí mismos acerca de ¿Quién soy yo? ¿̇Dónde estoy? ¿Qué voy a hacer? ¿Qué puedo hacer? ¿Qué quiero hacer? y ¿̇qué quiero ser? Cuando se les preguntó qué significó para ellos realizar su autobiografía, expresaron que fue un encuentro consigo mismo, que no se habían hecho preguntas sobre su lugar en el mundo tanto de orden personal como profesional, y que habían olvidado esos momentos que influenciaron su elección profesional, pero que es necesario recordar para mantener latente su motivación para ser profesor. Aquí se hizo evidente una autoimagen que relaciona su pasado, presente y futuro, pero es una autoimagen relacional, porque el sentido de aquello que hace lo justifica desde su experiencia vivida y de lo que quiere ser. Esto se constituyó en puntos de inspiración y entusiasmo para querer ser profesor.

En el segundo instrumento, la Entrevista Personal, el atributo que más se fortaleció fue el 1_narr (Figura 3). Atributo que fue el más recurrente en todos los instrumentos, y cobra sentido por cuanto los docentes noveles manifestaron que no se habían puesto a pensar ni a recordar momentos que influyeron en la decisión de ser profesor, pero que les ayudó a conocerse un poco más, a pensarse a sí mismos, a identificar sus cualidades y atributos que tienen como seres humanos y como profesores. Esto está en consonancia con lo que plantea Polkinghorne (1991), quien manifiesta que nos conocemos a nosotros mismos mediante un acto de conocimiento unificado de acciones y eventos del pasado junto a acciones y eventos futuros que anticipamos. Por otra parte, en la entrevista se manifestaron los seis atributos de IPD que se plantearon para el ámbito personal, sin embargo, no se identificó un número significativo de unidades de registro, lo que puede indicar que la entrevista restringe de alguna manera la mención de aspectos personales.

Y en el tercer instrumento, los Diarios de Profesor, el atributo que más unidades de registro codificó fue el número 5_Nomb (Figura 4) relacionado con el ejercicio práctico del profesor, que tal como lo plantean Bransford, Brown y Cocking, 1999; Leach y Scott, 2000; Prawat, 1992; Sparks y Hirsch, 1997, y Freeman, Marx y Cimellaro, 2004, manifiesta que el desarrollo y fortalecimiento del ejercicio práctico, desde lo que piensan y sienten los docentes, les permite reflexionar sobre su práctica, revisar y analizar sus conocimientos y hacer seguimiento de lo elaborado y practicado en su labor docente.

Expresiones manifiestas por los noveles docentes en sus escritos como "la autorreflexión" y "fui descubriendo" van revelando elementos de autoeficacia, referida esta, a las creencias personales acerca de las capacidades que posee para llevar a cabo nuevas acciones en su ejercicio profesional. Las creencias de autoeficacia pueden influir en el funcionamiento humano de muchas 
maneras, tal como lo manifiesta Bandura (1997). Estos procesos de reflexión, autoanálisis, co-análisis y discusiones permanentes promovidos desde la práctica profesional generan el fortalecimiento de su identidad profesional, por cuanto permiten un proceso de toma de decisiones y re-interpretaciones de valores de las experiencias de cada uno, con lo cual se consolidan de manera paulatina características de identidad, al mirarse a sí mismo y su función en el mundo. Tal como lo afirma $\mathrm{O}^{\prime}$ Connor (2008), el trabajo de los profesores radica en la participación emocional y en exigirse personalmente.

En los Diarios de Profesor, se manifestaron tres atributos deseables (3, 4 y 5 ) de los seis planteados para el ámbito personal, estos se relacionan con el proceso de autodescubrimiento, dado que establecen relaciones entre sus motivaciones personales y profesionales pasadas, presentes y futuras, y la experiencia vivida en sus escenarios de la práctica profesional, lo cual produjo nuevas actuaciones de manera consciente y reflexiva en su temprano ejercicio profesional.

Esta variedad de atributos fortalecidos se creó por las dinámicas desarrolladas en el marco del modelo de intervención docente que se diseñó, en las que se vincularon aspectos personales, profesionales y contextuales. Las dinámicas descritas antes promueven el desarrollo de la identidad profesional, puesto que esta se suscita por la interacción social que los profesores tienen en los contextos particulares en los que actúan, tal como lo señala Delahunty (2012).

De igual manera, se identificó que la IPD es construida, mantenida, entendida, negociada e interpretada a través del lenguaje, mediante el discurso que formulan sobre sí y sus relaciones, tal como lo manifiesta Bolívar (2006). Estos aspectos fueron evidentes a través de las elaboraciones escritas, discusiones y socializaciones realizadas, lo que produjo que este joven docente se convirtiera en un examinador consciente, riguroso y proactivo de cada uno de los factores que conforman el sistema educativo en aras de mejorarlo, desde lo que está a su alcance.

Este tipo de modelos de intervención con los docentes, en el que se movilizan sentimientos, conocimientos y acciones sobre un mismo objeto de conocimiento, es un potenciador que permite reconfigurar la IPD. Parte del reconocimiento de sí mismo y de su lugar en el mundo, y el rol profesional que desempeña, lo que hace que el pensar y el actuar en torno a la enseñanza se vayan transformando como producto de la reflexión sobre su práctica, al revisar y analizar sus conocimientos y actuaciones, acompañado de un grupo de pares y expertos, dinámica vivida en la práctica profesional.

\section{Reflexiones finales}

Los espacios académicos de la práctica profesional docente han de constituirse en escenarios de desarrollo profesional con profesores en formación inicial, en donde, a través del diseño de modelos de intervención auténticos, se contribuye a 
la reconfiguración de la identidad profesional docente, desde cada uno de los Ámbitos del Conocimiento Profesional del Profesor (ACPP) y en particular el ámbito personal. Esto en razón de que la discusión, reflexión y razonamiento permanente de las formas de pensar, sentir y actuar de los profesores revelan características de IPD fundamentales para querer pertenecer a un colectivo de profesores que se reconocen e identifican como tal, en pro de mejoras de la educación.

En exploración y el desarrollo de cada uno de los ACPP para la reconfiguración de la identidad profesional docente, fue necesario hacer estos ámbitos conscientes y explícitos a los profesores en formación durante toda la intervención. A través de las reflexiones, tanto individuales como colectivas, se les permitió mirarse a sí mismos, en relación con los otros y con el contexto. Este tipo de experiencias no solo les brindó a los profesores posibilidades de revisar y estructurar lo que sabían, sino que reconocieron que esto es solo una parte del entramado de conocimientos que ha de poseer como profesor.

En cuanto al ámbito personal, nunca lo habían explorado en su proceso de formación. Este ámbito — si bien no le resta importancia a los otros- es uno de los aportes más valiosos de la investigación, fue el de mayor influencia en el proceso de configuración y reconfiguración de la IPD. Se generaron experiencias para recrear momentos de su vida pasada y presente en los profesores noveles y ver cómo se proyectan hacia el futuro. La vinculación de aspectos personales, motivaciones familiares, sensibilidad con lo social y las relaciones establecidas con el momento sociocultural vigente crea un nuevo sentido de la profesión, al propiciar un conocimiento personal, de sus alcances y proyecciones. Esta experiencia transformó su función como examinador crítico de la sociedad y del sistema educativo.
La conformación de una comunidad de desarrollo profesional, que se reconoció bajo una misión en la que los profesores en formación inicial se involucraron emocional y cognitivamente, constituyó un escenario enriquecedor en el que los docentes, a través de un espacio común compartido, se reconocieron a sí mismos al identificar las cualidades personales y profesionales que tenían para ser docentes así como las habilidades que debían mejorar, producto de compartir experiencias con sus otros compañeros de la CODEP.

Las contribuciones de la comunidad a la IPD se instalan debido a la existencia de una afinidad o consonancia entre los principios de la misión de la CODEP y los profesores en formación. Pertenecer a un grupo o comunidad significa estar unidos bajo un conjunto de características e intereses comunes, que son compartidos por todos. Desde allí, ya se inicia con esta identificación y diferenciación de querer y ser profesor.

De igual manera, la CODEP se constituyó en escenario de formación permanente, puesto que allí se propiciaron momentos de discusión, reflexión y transformación de concepciones y modos de proceder de los profesores. Mirarse a sí mismos, analizar las actitudes y actuaciones de los otros profesores, generar discusiones grupales en torno a temas como problemáticas cotidianas de la enseñanza y del aprendizaje, el desempeño docente y de cómo los ven sus compañeros implicaron en sí el desarrollo de este espacio de crecimiento constructivo para todos los miembros de la comunidad.

La experiencia de compartir situaciones positivas y negativas, producto de la práctica docente, les permitió ganar confianza, junto con el desarrollo de un discurso en el marco de la cordialidad y respeto. También lograron analizar, emitir apreciaciones y recomendaciones sobre el desempeño y actitudes docentes

.


en aras de mejorar el ejercicio profesional, en el que se vincularon aspectos personales, profesionales y contextuales.

Otro de los aportes de la investigación fue crear un modelo de intervención docente con profesores en formación inicial, dado que estos modelos están dirigidos para profesores en ejercicio. Por tanto, desde esta investigación se amplía el modelo para profesores en formación inicial: este grupo de profesores, a través de la CODEP, se convertirá en un escenario de integración, articulación y movilización de saberes propios de la docencia y de las disciplinas en particular. A través de procesos de interacción, discusión, reflexión, transferencia, colaboración y transformación, le otorgaron un nuevo valor y sentido a la profesión docente.

En conclusión, para que la imagen de profesor se transforme en un contexto como el colombiano, y que sea una profesión apetecible y de reconocimiento social, se hace necesario generar lineamientos de política de formación docente que consideren la identidad profesional como un proceso consciente e intencionado y que haga parte de los currículos de los programas de formación docente en el país. Esto, de tal manera, que las nuevas generaciones de profesores identifiquen qué es lo que reconoce y diferencia a un profesor y posicionar su rol en los ámbitos social, político, cultural y financiero.

\section{Referencias}

Bandura, A. (1997). Self-efficacy: The exercise of control. New York: W.H. Freeman and Company

Beijaard, D., Meijer, P. C., y Verloop, N. (2004). Reconsidering research on teachers' professional identity. Teaching and Teacher Education, 20 (2), 107-128. https:// doi.org/10.1016/j.tate.2003.07.001

Bolívar, A. (2006). Identidad profesional del Profesorado de secundaria: Crisis y reconstrucción. Málaga, España: Aljibe.

Bransford, J. D., Brown, A., y Cocking, R. (1999). How people learn: Mind, brain, experience, and school. Washington, DC: National Research Council.

Bullough, R. V. (1997). Practicing theory and theorizing practice in teacher education. En J. Loughran y T. Russell (Eds.), Purpose, passion and pedagogy in teacher education (pp. 13-31). London/Washington, DC: Falmer Press.

Chong, S., Ling, L., y Chuan, K. (2011). Emerging professional teacher identity of pre-service teachers. Australian Journal of Teacher Education, 36 (8), 50-64.

Connelly, F. M., y Clandinin, D. J. (1996). Teachers' professional knowledge landscapes: Teacher stories-stories of teachers-school stories-stories of school. Educational Researcher, 25 (3), 24-30.

Clarke, D., y Hollingsworth, H. (2002). Elaborating a model of teacher professional growth. Teaching and teacher education, 18 (8), 947-967. 
Costa, A. L., y Garmston, R. J. (1994). Cognitive coaching: A foundation for renaissance schools. Washington: Christopher-Gordon Publishers.

Delahunty, J. (2012). "Who am I?": Exploring identity in online discussion forums. International Journal of Educational Research, (53), 407-420. doi:10.1016/i. ijer.2012.05.005

Freeman, J. G., Marx, R. W., y Cimellaro, L. (2004). Emerging considerations for professional development institutes for science teachers. Journal of Science Teacher Education, 15 (2), $111-131$.

García-Martínez, A. e lzquierdo, A. (2014). Contribución de la Historia de las Ciencias al desarrollo profesional de docentes universitarios. Enseñanza de las Ciencias, 32 (1), 265-281.

Gilmore, J., Hurst, M. y Maher, M. (2009). Professional identity development in teachers of science, technology, engineering, math, and science and math education. Proceedings of the NARST 2009 Annual Meeting. Recuperado de https://files. eric.ed.gov/fulltext/ED509408.pdf

Glickman, C. D. (1990). Pushing school reform to a new edge: The seven ironies of school empowerment. Phi Delta Kappan, 72 (1), 68-75.

Hong, J. Y. (2010). Pre-service and beginning teachers' professional identity and its relation to dropping out of the profession. Teaching and teacher Education, 26 (8), 1530-1543.

Izquierdo, M., García-Martínez, A., Quintanilla, M. y Adúriz-Bravo, A. (2016). Historia, filosofía y didáctica de las ciencias: Aportes para la formación del profesorado de ciencias. Bogotá: Universidad Distrital Francisco José de Caldas. http://die. udistrital.edu.co/sites/default/files/doctorado_ud/publicaciones/historia_filosofia_y_didactica_de_las_ciencias_aportes_para_la_formacion_del_profesorado_de_ciencias.pdf

Luehmann, A. L. (2007). Identity development as a lens to science teacher preparation. Science Education, 91 (5), 822-839.

O'Connor, K. E. (2008). "You choose to care": Teachers, emotions and professional identity. Teaching and teacher education, 24 (1), 117-126.

Knowles, G. J. (1992). Models for understanding pre-service and beginning teachers' biographies: Illustrations from case studies. En I. F. Goodson (Ed.), Studying teachers' lives (pp. 99-152). London: Routledge.

Kompf, M., Bond, W. R., Dworet, D., y Boak, R. T. (Eds.) (1996). Changing research and practice: Teachers' professionalism, identities and knowledge. London, Washington, DC: Falmer Press.

Polkinghorne, D. (1991). Narrative and self-concept. Journal of Narrative and Life History. $7(2-3), 135-153$. 
Prawat, R. S. (1992). Teachers' beliefs about teaching and learning: A constructivist perspective. American journal of education, 100 (3), 354-395.

Schepens, A., Aelterman, A., y Vlerick, P. (2009). Student teachers' professional identity formation: between being born as a teacher and becoming one. Educational Studies, 35 (4), $361-378$.

Sparks, D., y Hirsch, S. (1997). A new vision for staff development. Alexandria, VA, and Oxford, $\mathrm{OH}$ : Association for Supervision and Curriculum Development and National Staff Development Council.

Triantafyllaki, A. (2010). Performance teachers' identity and professional knowledge in advanced music teaching. Music Education Research, 12 (1), pp. 71-87.

\section{Para citar este artículo}

Aristizábal, A. (2019). Fortalecimiento de la identidad profesional docente en el ámbito personal del profesor. Revista Tecné, Episteme y Didaxis: TED, 46, 189-204. 